\title{
Case effects in letter-name matching: A qualitative visual field difference
}

\author{
JOSEPH B. HELLIGE and RON WEBSTER \\ University of Southern California, Los Angeles, California 90007
}

\begin{abstract}
Subjects indicated whether or not two letters presented simultaneously to the left or right visual field had the same name. On same-name trials, reaction time was faster when both letters had the same case (physically identical, or PI, pairs) than when they were of different case (name identical, or NI, pairs) and reaction time was faster for left visual field presentations than for right visual field presentations. For left visual field presentations, the letter-case effect was as large on different-name trials as on same-name trials, indicating that the NI-PI difference was not simply a physical identity effect. In contrast, for right visual field presentations, the letter-case effect was restricted to same-name trials. These qualitatively different patterns for the two visual fields are discussed in terms of hypothesized consequences of cerebral hemisphere asymmetry for information processing in the normal brain.
\end{abstract}

Because stimuli presented to one visual half-field project directly to the contralateral cerebral hemisphere, visual half-field differences have been useful for studying cerebral hemisphere asymmetry. One frequently used paradigm is based on a letter matching task popularized by Posner and his colleagues (e.g., Posner, Boies, Eichelman, \& Taylor, 1969; Posner \& Mitchell, 1967). In a typical experiment using this task, subjects are asked to indicate whether or not two visually presented letters have the same name. Two letters that have the same name may also have the same case (often referred to as physically identical, or PI, pairs, such as AA) or may have different cases (often referred to as name identical, or NI, pairs, such as Aa). The typical finding is that the reaction time (RT) to PI pairs is faster than the RT to NI pairs when the letters occur simultaneously or within about $1 \mathrm{sec}$ of each other. The typical explanation of this NI-PI difference is that PI trials permit a direct comparison of physical features that is more rapid than the extraction and comparison of letter names that are required on NI trials.

It has been hypothesized that, in right-handed humans, the right cerebral hemisphere is the more efficient for visuospatial processing and the left cerebral hemisphere is the more efficient for aspects of verbal processing (e.g., Cohen, 1977; Hellige, 1980a; Madden \& Nebes, 1980). As a result, it has been predicted that RT to PI pairs will be faster when the letters are projected to the left visual field/right hemisphere (LVF-RH) than to the right visual field/left hemisphere (RVF-LH);

The research reported here was supported in part by a grant to the first author from the National Science Foundation (BNS79-24445). Requests for reprints should be addressed to Joseph B. Hellige, Department of Psychology, University of Southern California, University Park, Los Angeles, California 90007. that is, there is an LVF-RH advantage. The opposite visual field advantage has been predicted for NI pairs. Several visual laterality experiments have produced results consistent with these predictions (e.g., Cohen, 1972; Davis \& Schmit, 1973; Geffen, Bradshaw, \& Nettleton, 1972), but there have been exceptions as well.

For example, Lefton and Haber (1974) found no visual field differences for PI pairs and either no visual field difference or an LVF-RH advantage for NI pairs (a result opposite to one of the above predictions). Hellige $(1975,1976)$ found that, at least under certain viewing conditions, the visual field difference for NI pairs depends on the amount of practice subjects have had on the task and on the perceptual quality of the stimuli. Specifically, extremely low levels of practice and perceptual degradation of the stimuli favor an LVF-RH advantage for NI pairs. Ledlow, Swanson, and Kinsbourne (1978) found no visual field difference for PI pairs and found an RVF-LH advantage for NI pairs only when PI and NI pairs were intermixed. Wilkens and Stewart (1974) used successive presentation of two letters and found no visual field differences in RT for either PI or NI pairs, but they did report small accuracy differences consistent with the earlier predictions. In a task involving successive presentation and matching of letter pairs (rather than single letters), Kroll and Madden (1978) actually found an RVF-LH RT advantage for PI pairs and an LVF-RH advantage for NI pairs among subjects with verbal Scholastic Aptitude Test (SAT) scores of at least 500 , which is not unusually high for college populations.

In light of these diverse findings, the present experiment was conducted to examine further the effects of case identity in letter-name matching using lateralized stimulus presentation. One purpose was to compare any laterality effects for two experimental sessions 
(i.e., to further examine any practice effects). A second purpose was to determine whether letter-case identity effects are restricted to same-name trials when the letters are presented briefly to the visual periphery. The popular interpretation of the NI-PI difference discussed earlier predicts that, on different-name trials, RT should not depend on whether the two letters have the same case (because even when the cases are the same, the letters are not physically identical). Indeed, Posner et al. (1969) report case identity to be irrelevant on different-name trials when the letters are presented to the fovea for relatively long durations. However, the laterality studies that have used these tasks have not shown that the same is true under the more difficult viewing conditions necessary in laterality studies. Of the experiments cited earlier, only Kroll and Madden (1978) even indicate that case effects were examined on different-name trials. Interestingly, among their subjects with verbal SAT scores of at least 500 , the advantage for same-case pairs was almost as large for different-name pairs as for same-name pairs on LVF-RH trials, whereas on RVF-LH trials, the advantage for same-case pairs was restricted to same-name pairs.

\section{METHOD}

\section{Apparatus}

The subject sat at a table facing a $45 \times 30 \mathrm{~cm}$ dark gray screen approximately $50 \mathrm{~cm}$ away at eye level. On top of the table were two telegraph keys mounted about $40 \mathrm{~cm}$ apart and equidistant from the center of the viewing screen. Above one key was a card labeled "S," and above the other key was a card labeled "D." A Gerbrands three-field projection tachistoscope (Model G1176) was used to rear-project the fixation dot and letter stimuli onto the screen. Stimulus presentation parameters were generated by a Gerbrands six-channel timer (Model 300-6T), and response latency was measured by a Gerbrands digital clock/counter (Model G1270). Summary statistics for each experimental session were computed by an Apple II microprocessor.

\section{Stimulus Materials}

The stimuli consisted of the letters A, B, D, E, G, H, Q, and $R$, in both upper- and lowercase form (photographed as negatives from Cello-Tak Helvetica 5209-C and 5209-L, respectively). These letters were chosen because their upperand lowercase forms are not physically similar. When presented to the subject, the letters appeared as white letters on a dark background and the two letters of a pair appeared one above the other, with about $.5 \mathrm{deg}$ of visual angle between the two letters of the pair. Uppercase letters spanned between 1.6 and $2.2 \mathrm{deg}$ of visual angle in width and between 2.2 and $2.5 \mathrm{deg}$ in height. Lowercase letters spanned between .9 and $1.6 \mathrm{deg}$ in width and between 1.6 and $2.2 \mathrm{deg}$ in height. Average luminance of the letters was about $1.1 \mathrm{~cd} / \mathrm{m}^{2}(.3 \mathrm{fL})$. The center of the projected letters averaged $6.6 \mathrm{deg}$ of visual angle to the left or right of the center of the screen with a standard deviation of $.1 \mathrm{deg}$ of visual angle. A white fixation dot spanned .2 deg of visual angle when projected on the screen and had a luminance of about $.33 \mathrm{~cd} / \mathrm{m}^{2}$ (.1 fL).

For convenience, the upper letter of each pair was projected via one channel of the tachistoscope and the lower letter was projected simultaneously via a different channel. Such an arrangement increases the number of different pairings that can be created from a given number of slide stimuli. It should be noted that left and right visual field trials were presented equally often by both tachistoscope channels: that is, visual field was not confounded with channels. Stimulus sequences were constructed so that the upper- and lowercase forms of each of the eight letters occurred twice within each visual field for each set of 32 trials. In addition, each set of 32 trials contained four occurrences of each of eight trial types defined by the orthogonal combination of (1) same vs. different letter names, (2) same vs. different letter cases, and (3) right vs. left visual field. These trial types were arranged randomly, with the restriction that there be no more than four consecutive occurrences of either same-name or different-name pairs.

\section{Procedure}

Each subject participated in two experimental sessions on different days within the same week. On each day, the subject received 10 blocks of 32 trials each. The 320 trials/day consisted of 40 occurrences of each of the eight trial types described above. Half of the subjects indicated "same" responses with their right hand and "different" responses with their left hand, and the remaining subjects had the opposite response arrangement.

At the beginning of the experiment, each subject was instructed to keep the index finger of the right hand on the right telegraph key and the index finger of the left hand on the left telegraph key throughout the experimental session. The subjects were told to fixate their gaze on the central fixation dot when it appeared and to maintain that fixation until after they responded to the letter pair. The subjects were then told that on each trial a letter pair would occur in the left or right visual field $1 \mathrm{sec}$ after the fixation dot appeared, with visual field determined randomly, so that the best strategy was to fixate on the central dot rather than on either side. Subjects were instructed to press the key labeled " $S$ " if the two letters on a trial had the same name and the key labeled " $D$ " if the two letters had different names. Subjects were further asked to respond as quickly and as accurately as possible. They were also informed that the two letters would sometimes be of different cases but that case was irrelevant to their response. At the beginning of the first experimental session, subjects were given 12 practice trials to make sure they understood the task.

At the beginning of each trial, the fixation dot appeared in the center of the screen. After $1 \mathrm{sec}$, the dot disappeared and, simultaneously, a letter pair appeared for $10 \mathrm{msec}$. The intertrial interval within each 32-trial block was $6 \mathrm{sec}$, and the time between blocks was about $15 \mathrm{sec}$.

\section{Subjects}

Ten men and 10 women volunteers from introductory psychology courses at the University of Southern California participated in the experiment. All subjects had normal or corrected-to-normal visual acuity in both eyes, were native speakers of English, and were strongly right-handed according to a shortened version of the Edinburgh Handedness Inventory (Oldfield, 1971).

\section{RESULTS}

A preliminary analysis indicated no reliable effects related to subjects' sex, so the results are collapsed across men and women. The error rates were very low (7.1\% on Day 1 and $4.2 \%$ on Day 2), precluding a meaningful statistical analysis. Accordingly, emphasis is placed on the RT of correct responses as the primary dependent variable. The .05 level of statistical significance is used throughout the present article.

For each subject, the median RT of correct responses was determined for each of the eight trial types on each 


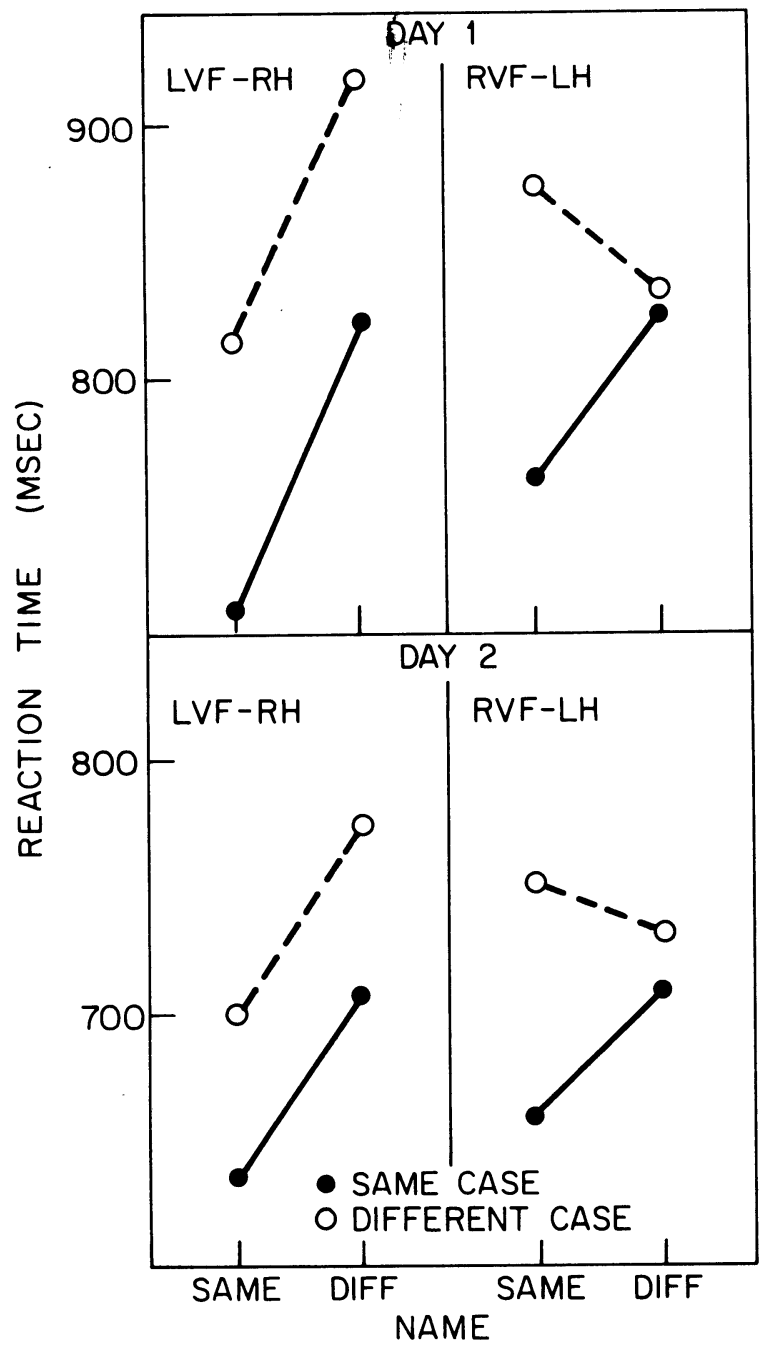

Figure 1. Reaction time in milliseconds for same-name and different-name letter pairs. The results for Days 1 and 2 are shown in the upper and lower panels, respectively, and the results for LVF-RH and RVF-LH trials are shown in the left and right panels, respectively. The parameter in each panel is same vs. different case.

of the 2 days of the experiment. Figure 1 shows the mean of median RTs for both same-name and differentname letter pairs. The results for LVF-RH and RVF-LH trials are shown in the left and right panels, respectively, and the results for Days 1 and 2 are shown in the upper and lower panels, respectively. The parameter in each panel is same-case pairs vs. different-case pairs. There was a large decrease in RT from Day 1 to Day 2 $[\mathrm{F}(1,19)=24.14, \mathrm{MSe}=39,709]$, but the pattern of results was identical for both days. Consequently, the remaining results ignore the Day 1 vs. Day 2 variable.

Collapsed across visual field, RT was faster to samename pairs than to different-name pairs $[\mathrm{F}(1,19)=$ $12.79, \mathrm{MSe}=18,144]$ and faster to same-case pairs than to different-case pairs $[\mathrm{F}(1,19)=76.23$, MSe $=5,422]$. The case effect was larger for same-name pairs than for different-name pairs, producing a significant Name by Case interaction $[F(1,19)=7.36, \mathrm{MSe}=6,223]$. However, the case effect was not completely absent for different-name pairs, as reported under other conditions (e.g., by Posner et al., 1969). Furthermore, the effects just noted were not the same for LVF-RH trials and RVF-LH trials, producing the following significant interactions: Name by Visual Field $[\mathrm{F}(1,19)=15.23$, $\mathrm{MSe}=7,483]$, Case by Visual Field $[\mathrm{F}(1,19)=5.67$, $\mathrm{MSe}=2,766]$, and Name by Case by Visual Field $[\mathrm{F}(1,19)=10.42, \mathrm{MSe}=3,631]$. To shed light on the nature of these interactions, separate analyses were performed on the results from each visual field.

As shown in the left-hand panels of Figure 1, on LVF-RH trials RT was faster to same-name pairs than to different-name pairs $[F(1,19)=19.81, \mathrm{MSe}=16,944]$ and faster to same-case pairs than to different-case pairs $[F(1,19)=73.00, \mathrm{MSe}=4,042]$. However, the case effect was as large for different-name pairs as for samename pairs, producing no Name by Case interaction $(\mathrm{F}<1.0)$ (cf. Kroll \& Madden, 1978).

The results were quite different on RVF-LH trials, as shown in the right-hand panels of Figure 1. There was no overall RT difference between same-name and different-name pairs $[F(1,19)<1.0]$, and the overall case effect was present but smaller than it was on LVF-RH trials $[\mathrm{F}(1,19)=32.31, \mathrm{MSe}=4,147]$. The most striking difference between the visual fields was the highly significant Name by Case interaction on RVF-LH trials $[\mathrm{F}(1,19)=16.80, \mathrm{MSe}=4,968]$. As shown in Figure 1, on RVF-LH trials the case effect was large for same-name pairs but virtually absent for different-name pairs.

\section{DISCUSSION}

On same-name trials, RT was consistently faster for PI pairs than for NI pairs, a rather typical result for this paradigm. There was a small, but consistent, LVF-RH RT advantage averaging about $40 \mathrm{msec}$ for both PI and NI pairs, producing a statistically significant visual field effect for same-name pairs $[\mathrm{F}(1,19)=5.54, \mathrm{MSe}=1,083]$. For PI pairs, Moscovitch (1979) has noted that an LVF-RH advantage has been found primarily in those studies that have used very brief exposure durations or perceptually degraded stimuli. For NI pairs, Hellige (1976) has also found that using conditions of reduced perceptual quality favors an LVF-RH advantage. In the present experiment, the brief exposure duration of $10 \mathrm{msec}$ and the relatively large deviation of the stimuli from the center of vision $(6.6 \mathrm{deg}$ off center) produced impaired viewing conditions relative to most of the visual laterality studies cited in the introduction. Perhaps it is this perceptual impairment that produces the consistent LVF-RH advantage for same-name trials.

In the present experiment, the interpretation of the difference in RT to NI and PI pairs is beclouded by the presence of a case effect for different-name trials. As noted earlier, even when the two letters in a different-name pair have the same case, they are not physically identical. Therefore, at least for LVF-RH trials, it cannot be argued that the NI-PI difference is simply a physical identity effect, although such an argument is not unreasonable for RVF-LH pairs. It is possible that the case effect on different-name trials that was found here and that can be seen for some subjects in Kroll and Madden (1978) is 
not unusual with lateralized stimulus materials briefly presented. Unfortunately, the other studies referred to in the introduction do not present any information about possible case effects for different-name trials, so it is difficult to specify the conditions under which they might be most likely to occur. Nevertheless, the qualitatively different pattern of effects for the two visual fields in the present experiment suggests that the NI-PI differences may be produced by somewhat different mechanisms for LVF-RH and RVF-LH trials.

It is instructive to consider the types of processing that might produce the pattern of results found for each visual field. The presence of a case effect for different-name trials suggests some sort of initial analysis of case identity. For the particular letters used in this experiment, upper- and lowercase letters might be partially distinguished on the basis of gross physical characteristics such as size, density, overall letter envelope, etc. With very brief, nonfoveal presentations, it may become efficient to first determine whether the overall, global characteristics of the letters are the same (i.e., whether they have the same case). If so, then an analysis of more specific features could determine unambiguously whether or not the letters had the same name. However, if the cases are different, something more complex (and, presumably, more time-consuming) would have to be done. Note that this sort of initial global case analysis would produce a case identity advantage for both same-name and different-name trials and would be consistent with the results on LVF-RH trials. In contrast, if processing begins with a more specific analysis of feature identity, then one might expect an advantage for PI pairs, but no case effect for different-name pairs. This is the type of model most frequently proposed for this paradigm and is more consistent with the results on RVF-LH trials.

Although the preceding discussion of alternative processing mechanisms is speculative, it is consistent both with the present results and with other recent discussions of qualitative processing differences related to visual field. For example, in two memory search experiments, Hellige $(1980 \mathrm{~b})$ reported that perceptual degradation of a probe letter increased the slope of the memory set-size function when the probe was presented to the LVF-RH but not when the probe was presented to the RVF-LH. Hellige suggested that when the probe was processed initially by the right cerebral hemisphere, there was a tendency to use a literal encoding of the probe (which retained the effects of degradation) to perform a relatively visuospatial memory comparison. In contrast, when the probe was processed initially by the left cerebral hemisphere, there was a tendency to rely on a more abstract encoding from which the effects of perceptual degradation were removed. Such results are consistent with other evidence suggesting that the right and left hemispheres are biased toward global or visuospatial and analy tic or verbal modes of processing, respectively (e.g., Cohen, 1977; Hellige, 1980a; Madden \& Nebes, 1980). In a series of face recognition studies with normal adults, Sergent (in press) reports that on LVF-RH trials, there was a tendency to process the facial features in their order of perceptual salience, whereas on RVF-LH trials, there was a tendency to scan the features serially from top to bottom, regardless of salience. Sergent concludes that the right cerebral hemisphere typically begins by processing the most salient features of visual stimuli, whereas the left cerebral hemisphere is more consistently analy tic and serial in approach.

In view of these other results, it is not particularly surprising that the pattern of results in the present experiment was qualitatively different for LVF-RH and RVF-LH trials. Furthermore, the suggestions made earlier about the nature of processing on LVF-RH and RVF-LH trials in the present experiment are conceptually similar to the different models proposed by Hellige (1980b) and Sergent (in press) using very different paradigms. The examination of additional qualitative visual field differences will be useful to pinpoint more precisely the nature of cerebral hemisphere asymmetry and the implications for information processing in the normal brain.

\section{REFERENCES}

Conen, G. Hemispheric differences in a letter classification task. Perception \& Psychophysics, 1972, 11, 139-142.

Conen, G. The psychology of cognition. London: Academic Press, 1977.

DAvis, R., \& Schmit, V. Visual and verbal coding in the interhemispheric transfer of information. Acta Psychologica, 1973, 37, 229-240.

Geffen, G., Bradshaw, J. L., \& Nettleton, N. C. Hemispheric asymmetry: Verbal and spatial encoding of visual stimuli. Journal of Experimental Psychology, 1972, 95, 25-31.

Hellige, J. B. Hemispheric processing differences revealed by differential conditioning and reaction time performance. Journal of Experimental Psychology: General, 1975, 104, 309-326.

Hellige, J. B. Changes in same-different laterality patterns as a function of practice and stimulus quality. Perception \& Psychophysics, 1976, 20, 267-273.

Hellige, J. B. Cerebral hemisphere asymmetry: Methods, issues, and implications. Educational Communication and Technology, 1980, 28, 83-98. (a)

Hellige, J. B. Effects of perceptual quality and visual field of probe stimulus presentation on memory search for letters. Journal of Experimental Psychology: Human Perception and Performance, 1980, 6, 639-651. (b)

Kroll, N. E. A., \& MadDen, D. J. Verbal and pictorial processing by hemisphere as a function of the subject's verbal Scholastic Aptitude Test score. In J. Requin (Ed.), Attention and performance VII. Hillsdale, N.J: Erlbaum, 1978.

Ledlow, A., Swanson, J. M., \& Kinsbourne, M. Reaction times and evoked potentials as indicators of hemispheric differences for laterally presented name and physical matches. Journal of Experimental Psychology: Human Perception and Performance, 1978, 4, 440-454.

Lefton, L. A., \& HABER, R. N. Information extractions from different retinal locations. Journal of Experimental Psychology, 1974, 102, 975-980.

Madden, D. J., \& Nebes, R. D. Visual perception and memory. In M. C. Wittrock (Ed.), The brain and psychology. New York: Academic Press, 1980.

Moscovitch, M. Information processing and the cerebral hemispheres. In M. S. Gazzaniga (Ed.), Handbook of behavioral neurobiology (Vol. 2). New York: Plenum, 1979.

Oldfield, R. C. The assessment of handedness: The Edinburgh inventory. Neuropsychologia, 1971, 9, 97-111.

Posner, M. I., Boies, S. I., Eichelman, W. H., \& Taylor, R. I. Retention of visual and name codes of single letters. Journal of Experimental Psychology Monograph, 1969, 79(1, Pt. 2).

Posner, M. I., \& Mitchell, R. F. Chronometric analysis of classification. Psychological Review, 1967, 74, 392-409.

SERGENT, J. About face: Left hemisphere involvement in processing physiognomies. Journal of Experimental Psychology: Human Perception and Performance, in press.

Wilkens, A., \& Stewart, A. The time course of lateral asymmetries in visual perception of letters. Journal of Experimental Psychology, 1974, 102, 905-908.

(Received for publication March 31, 1981.) 